\title{
Arytenoid Muscle
}

National Cancer Institute

\section{Source}

National Cancer Institute. Arytenoid Muscle. NCI Thesaurus. Code C32149.

A muscle that arises from the arytenoid cartilage of one side of the larynx and inserts to the arytenoid cartilage of the opposite site. 